\title{
Prenatal antidepressant use and risk of attention-deficit/hyperactivity disorder in offspring: population based cohort study
}

\author{
Kenneth K C Man, ,2,3,4 Esther W Chan, ${ }^{1}$ Patrick Ip, ${ }^{2}$ David Coghill,,5,6 Emily Simonoff,7 Phyllis K L Chan, ${ }^{8}$ \\ Wallis CY Lau, ${ }^{1}$ Martijn J Schuemie, ${ }^{9}$ Miriam C J M Sturkenboom, ${ }^{4}$ lan C K Wong ${ }^{1,2,3}$
}

For numbered affiliations see end of article.

Correspondence to: I C K Wong, Research Department of Practice and Policy, UCL School of Pharmacy, London WC1N 1AX,UKi.wong@ucl.ac.uk Additional material is published online only. To view please visit the journal online.

Cite this as: BMJ 2017;357:j2350 http://dx.doi.org/10.1136/bmj.j2350

Accepted: 11 May 2017

\section{ABSTRACT}

OBJECTIVE

To assess the potential association between prenatal use of antidepressants and the risk of attentiondeficit/hyperactivity disorder (ADHD) in offspring.

DESIGN

Population based cohort study.

SETTING

Data from the Hong Kong population based electronic medical records on the Clinical Data Analysis and Reporting System.

\section{PARTICIPANTS}

190618 children born in Hong Kong public hospitals between January 2001 and December 2009 and followed-up to December 2015.

\section{MAIN OUTCOME MEASURE}

Hazard ratio of maternal antidepressant use during pregnancy and ADHD in children aged 6 to 14 years, with an average follow-up time of 9.3 years (range 7.4-11.0 years).

RESULTS

Among 190618 children, 1252 had a mother who used prenatal antidepressants. 5659 children (3.0\%) were given a diagnosis of ADHD or received treatment for ADHD. The crude hazard ratio of maternal antidepressant use during pregnancy was $2.26(\mathrm{P}<0.01)$ compared with non-use. After adjustment for potential confounding factors, including maternal psychiatric disorders and use of other psychiatric drugs, the adjusted hazard ratio was reduced to 1.39 (95\% confidence interval 1.07 to $1.82, \mathrm{P}=0.01$ ). Likewise, similar results were observed when comparing children of mothers who had used antidepressants before pregnancy with those who were never users $(1.76,1.36$

\section{WHAT IS ALREADY KNOWN ON THIS TOPIC}

Whether to prescribe drugs for depression during pregnancy is a complex decision Prenatal use of antidepressants is considered a risk factor for attention-deficit/ hyperactivity disorder (ADHD) in children, but evidence is inconclusive The negative consequences of untreated maternal depression might also affect childhood development

\section{WHAT THIS STUDY ADDS}

The risk of ADHD was similar between the offspring of mothers who used antidepressants during pregnancy and those who used before pregnancy only, whereas the risk was higher for offspring of mothers with psychiatric disorders irrespective of whether antidepressants were used

Evidence suggests that the association between prenatal antidepressant use and risk of ADHD may at least partially be explained by confounding by indication of antidepressants

If there was a causal association; then the size of the effect is probably smaller than what has been reported previously to 2.30, $\mathrm{P}<0.01)$. The risk of ADHD in the children of mothers with psychiatric disorders was higher compared with the children of mothers without psychiatric disorders even if the mothers had never used antidepressants $(1.84,1.54$ to $2.18, \mathrm{P}<0.01)$. All sensitivity analyses yielded similar results. Sibling matched analysis identified no significant difference in risk of ADHD in siblings exposed to antidepressants during gestation and those not exposed during gestation ( $0.54,0.17$ to $1.74, \mathrm{P}=0.30)$.

\section{CONCLUSIONS}

The findings suggest that the association between prenatal use of antidepressants and risk of ADHD in offspring can be partially explained by confounding by indication of antidepressants. If there is a causal association, the size of the effect is probably smaller than that reported previously.

\section{Introduction}

Attention-deficit/hyperactivity disorder (ADHD) is a common neurodevelopmental disorder in children and adolescents. It is characterised by pervasive hyperactivity, inattention, and impulsiveness, which impairs the lives of young people. ${ }^{1}$ ADHD is common among school aged children, with a worldwide prevalence of 5-7\%.23 Rates of diagnosis are high in North America and, while ADHD is under-diagnosed in most other parts of the world, rates of identified cases are increasing. ${ }^{2}$ Owing to early onset, lifelong persistence, and high levels of comorbidities and impairment associated with ADHD, 4 its negative impact on social outcomes, education, and the health of patients and their caregivers is substantial. ${ }^{5}$ Understanding the risk factors for ADHD is an important public health matter.

Recent studies have suggested a potential association between maternal prenatal use of antidepressants, in particular selective serotonin reuptake inhibitors (SSRIs), and the risk of ADHD in children. ${ }^{6-11}$ A systematic literature search identified six observational studies, including three cohort studies ${ }^{611}$ and three case-control studies, ${ }^{7810}$ which investigated the association between antidepressant use in pregnancy and ADHD in children, with the study populations from Scandinavian countries or North America. However, findings were inconsistent. -11 $^{-11}$ Only one case-control study $^{7}$ restricted its sample to children aged at least 5 years, whereas the others did not. ${ }^{6-11}$ As ADHD is usually diagnosed clinically after age 5 years, previous studies might have identified unrepresentative samples because of large numbers of children aged less than 5 years, leading to biased estimates of the actual risk. Furthermore, a controversial issues is whether the observed association between antidepressant use in pregnancy 
and ADHD in childhood is causal or may be confounded by other "third factor" variables, such as underlying maternal psychiatric disorder (confounding by indication). ADHD is highly heritable and often comorbid with other mental health problems, including anxiety and depression, both of which are commonly treated with antidepressants. ${ }^{12}$ The association between prenatal use of antidepressants and ADHD in offspring could therefore be confounded by maternal or paternal psychiatric disorders. ${ }^{713}$ A recently published study using information from a Swedish registry found an increased risk of ADHD in offspring with prenatal exposure to antidepressants using population wide analyses. However, the authors reported non-significant results in sibling matched analyses and recommended that future research should use designs to help account for unmeasured genetic and familial confounders to explore the associations with prenatal exposure to antidepressants in other countries. ${ }^{11}$ Studies to date have not adequately addressed the critical question of confounding by indication. We hypothesised that the apparent link between prenatal use of antidepressants, including SSRIs, and the increased risk of ADHD in offspring is at least partly confounded by maternal risk factors. Consequently, we assessed the association between maternal prenatal use of antidepressants and the risk of ADHD in offspring, and in particular the possibility of confounding by indication in this association by examining the effect of pre-existing maternal psychiatric illness.

\section{Methods}

\section{Data source and study design}

We carried out a cohort study nested in the electronic health record of the Clinical Data Analysis and Reporting System (CDARS), a territory wide database in Hong Kong. CDARS was developed by the Hong Kong Hospital Authority, a statutory body that manages all public hospitals and their associated ambulatory clinics in Hong Kong. The hospital authority health service is accessible to all Hong Kong residents (over 7 million). Data from CDARS have been used for various pharmacoepidemiological studies. ${ }^{14-18}$ The database contains patient specific data, including personal information, payment method, prescription information, pharmacy dispensing information, diagnosis, laboratory test results, and admission and discharge information. ${ }^{19}$ CDARS contains the records of all in-patient, out-patient, and emergency room admissions in hospital authority clinics and hospitals since 1995. Records are coded to protect patient confidentiality. A detailed description of CDARS can be found elsewhere. ${ }^{16}$

\section{Source population}

The source population included all children born in public hospitals in Hong Kong between 1 January 2001 and 31 December 2009. Therefore by 31 December 2015, when we collected the child outcome data, all children would have at least six years' follow-up. To avoid potential bias we excluded children without valid mother-child linkage, born to mothers who were non-Hong Kong residents and whose medical records were likely to be incomplete (18.8\%, 45831 out of 243526 children). Only live births were included in the analysis. We defined a valid mother-child linkage as an exact match of mother and child patient identification numbers, delivery date, and delivery hospital. The mother-child linkage is created by the hospital authority for clinical management, and the mother and child records are linked permanently immediately after delivery; hence this linkage is highly accurate.

\section{Pregnancy period and maternal antidepressant use} We identified prenatal antidepressant use in mothers with respect to the pregnancy period. The gestational age of pregnancy is directly recorded by healthcare professionals, and the last menstrual period was calculated by date of delivery minus gestational age at delivery. We defined the pregnancy period as the period between the last menstrual period and the date of delivery, with the date of the last menstrual period as the start of cohort entry, and we defined the time before the last menstrual period as prepregnancy. To examine any potential effects on the timing of antidepressant use, we further divided the pregnancy period into trimesters: first trimester, 0-90 days after the last menstrual period; second trimester, 91-180 days after the last menstrual period; and third trimester, 181 days after the last menstrual period to delivery.

Antidepressant use in mothers was extracted from the prescribing and dispensing records in CDARS. All drugs in the British National Formulary chapter 4.3 were included. We defined antidepressant use as the length of time using the drugs, and this was estimated by the length of time between start and end dates for each prescription as recorded in CDARS. When end dates for prescriptions were missing we used the median treatment duration to impute data. The rate of missing data is low, with $98.4 \%$ of antidepressant prescriptions having complete information about treatment duration. Children were considered to have been exposed prenatally during the respective risk window if maternal use of drugs overlapped with a time point in the pregnancy window. Based on maternal antidepressant use in different risk periods, we classified the children into four groups: those with mothers who used drugs before conception but who stopped treatment when pregnant (preconception users), or negative control group 1; those who never used drugs before and during pregnancy, or never users (in subgroup analyses we further classified this group into (a) never users without psychiatric disorders, and (b) never users with psychiatric disorders, or negative control group 2); those whose mothers were non-gestational users (a combined group of never users and preconception users); and those whose mothers used antidepressants during pregnancy (gestational users), or the active treatment group.

Through CDARS we also identified maternal psychiatric disorders diagnosed before and during pregnancy. We coded these using ICD-9-CM (international classification of diseases, ninth revision, clinical modification) diagnosis code 290-319. If the diagnosis date was recorded within a respective risk window, we considered the mother to have a psychiatric disorder. 


\section{Follow-up}

The follow-up period for all liveborn children started on the date of delivery and ended on the date of ADHD diagnosis, date of first drug prescription for ADHD, date of death, or 31 December 2015, whichever came first.

\section{Outcome definition}

Study outcomes in the liveborn children were an ADHD diagnosis, registered as ICD-9-CM diagnosis code 314, or a prescription for an ADHD drug, namely methylphenidate or atomoxetine (British National Formulary chapter 4.4), as recorded in CDARS, as these are the only available drugs for ADHD in Hong Kong.

\section{Covariates}

Data on maternal comorbidities and other drugs were obtained from CDARS. As with previous studies, ${ }^{6-10}$ covariates considered for confounding adjustment were maternal age at delivery, infant's sex, birth year, birth hospital, parity, maternal underlying medical conditions before delivery (pre-existing diabetes, epilepsy, gestational diabetes, psychiatric conditions, hypertension), use of other psychotropic drugs (antipsychotics, British National Formulary chapter 4.2.1, 4.2.2), and socioeconomic status.

\section{Statistical analysis}

We adopted several approaches for analysis. For the first we compared ADHD status of children whose mothers used antidepressants during pregnancy (gestational users) with children whose mothers did not (non-gestational users). Hazard ratios for the association between antidepressant use for each trimester and outcome were estimated using Cox proportional hazard regression models. Robust standard error was used to adjust for data clustering. We selected the previously described covariates and added them to analytical models.

As the likelihood of being treated with antidepressants might be higher in mothers with severe psychiatric disorders, we carried out three levels of adjustment to assess the effect of confounding by indication. The first model (model 1) included all the covariates mentioned except for maternal underlying psychiatric illness and other psychotropic drug use before and during pregnancy. Model 2 extended model 1 to include all maternal underlying psychiatric disorders. Model 3 added to model 2 by further adjusting for concurrent use of other psychotropic drugs. To investigate class effects we repeated the analyses and split the antidepressants into subgroups (SSRIs and non-SSRIs).

In another approach we explored the impact of confounding by indication. Firstly, we compared preconception users of antidepressants (women who stopped treatment before conception) with never users. We restricted the cohort to mothers who neither used antidepressants nor antipsychotics during pregnancy. Mothers who had only used antidepressants before pregnancy were defined as the preconception group, which acted as a negative control group 1 . An increased risk of ADHD in the offspring of mothers among the preconception group might indicate confounding by indication as the fetus was not exposed to antidepressants. Similarly, mothers with gestational use of antidepressants were compared with the preconception group to assess if there is a difference in risk of ADHD in children. Secondly, we assessed the association between maternal psychiatric disorders and risk of ADHD in children. The analysis was restricted to mothers with a psychiatric diagnosis who had never used antidepressants or antipsychotics before delivery (never users), this acted as negative control group 2. This allowed us to estimate the role of maternal psychiatric disorder in ADHD in their offspring. If a psychiatric disorder in mothers is associated with risk of ADHD in offspring, it is a confounder between prenatal use of antidepressants and risk of ADHD. As a psychiatric disorder is an indication for antidepressants, this introduces the possibility of confounding by indication. Thirdly, we restricted the analysis to mothers who used either antidepressants or antipsychotics during pregnancy. We repeated the analyses by comparing use of SSRIs with use of non-SSRIs, and antidepressants with antipsychotics using the method described previously. This analysis used the antipsychotic drug group as an active comparator group, to minimise the effect of confounding by indication.

Several additional sensitivity analyses were conducted to test the validity and robustness of the initial analyses. We carried out a sensitivity analysis by including children who were born after 1 January 2009 to investigate the potential impact of under-diagnosis, because the rate of ADHD diagnosis among younger children has increased in recent years. Further analyses were conducted based on different drug non-adherence scenarios. To assess the effect of exposure misclassification, we further extended each period of antidepressant use by adding 30 and 60 days to the end of each period. Children taking drugs for ADHD but without an ADHD diagnosis were removed from the analysis. We restricted the analyses to the first pregnancy only. This was to investigate the potential clustering effect of children who were born to the same mother. We conducted an additional sensitivity analysis by adjusting only for maternal depression, anxiety, and substance abuse disorder in our model. As ADHD is commonly diagnosed in children around the age of 5 years, we conducted a sensitivity analysis by starting the follow-up time in children at age 5 years. Children who had a diagnosis before age 5 were removed from this analysis.

We also conducted a post hoc sibling matched analysis, a design that has been used previously to evaluate the association between smoking during pregnancy and risk of hyperkinetic disorder (a severe form of ADHD). ${ }^{20}$ This analysis was used to control for shared genetic and social confounding. We used stratified Cox regression with a separate stratum for each family identified by the mother's unique identification number. In the stratified Cox regression model, each family has its own baseline rate function reflecting the family's shared genetic and social factors. The stratified Cox regression model is an extension of the paired binomial model, taking into account the differences in follow-up 
time. Thus, only sibling pairs discordant for maternal antidepressant use and ADHD were able to contribute information to the estimates.

A significance level of 5\% was used in all statistical analyses. Data manipulation and analysis were conducted through Microsoft Excel and Statistical Analysis System (SAS) v9.3 (SAS Institute, Cary, NC). KM and WL carried out the analyses in parallel and independently. They cross checked the programming and results for accuracy and consistency.

\section{Patient involvement}

No patients were involved in setting the research question or the outcome measures, nor were they involved in developing plans for design or implementation of the study. No patients were asked to advise on interpretation or writing up of results. There are no plans to disseminate the results of the research to study participants or the relevant patient community.

\section{Results}

The analysis included 190618 pairs of mother-child records (fig 1). The mean maternal age at delivery was 31.2 years (SD 5.1 years, table 1). Overall, 1252 children were exposed to maternal antidepressants during gestation. Among their mothers, 425 and 470 received SSRI and non-SSRI antidepressant monotherapy, respectively, 129 received SSRIs and non-SSRIs, 101 received SSRIs and antipsychotics, 91 received non-SSRIs and antipsychotics, and 36 received SSRIs, non-SSRIs, and antipsychotics. Of 2275 mothers who received antidepressants before pregnancy; 1486 had discontinued treatment before pregnancy and 789 had continued treatment into pregnancy (see supplementary material 1 and 2).

In this cohort, 5659 children had a diagnosis of ADHD or received a drug for ADHD. The mean follow-up time was 9.28 years (range 7.4-11.0 years). The crude hazard ratio of antidepressant use during pregnancy and ADHD was 2.26 when gestational users were compared with non-gestational users. The estimate was similar in model 1 (adjusted hazard ratio 2.39, 95\% confidence interval 1.90 to 3.02 ) but reduced to 1.41 (1.08 to1.83) and

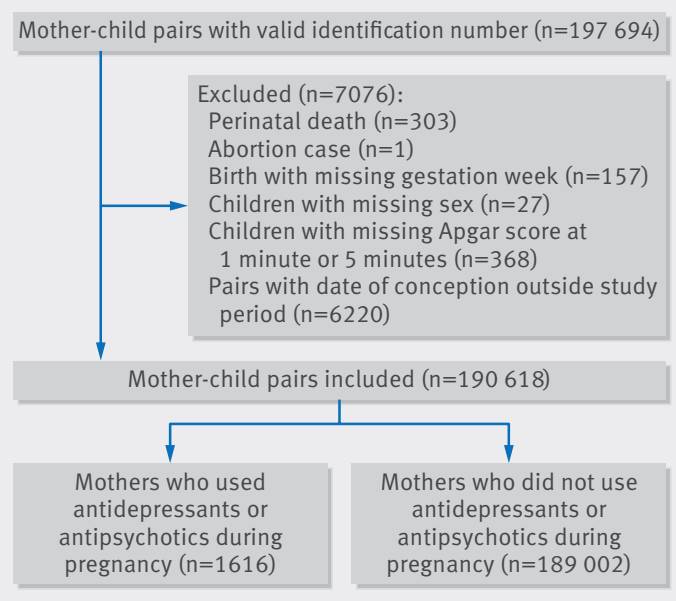

Fig 1 | Flowchart of mother-child pairs identification
1.39 (1.07 to 1.82) in models 2 and 3, respectively, when maternal psychiatric disorder and additional psychotropic drugs were included. The corresponding hazard ratios in the three trimesters were similar; the fully adjusted hazard ratios (model 3) were 1.43 (95\% confidence interval 1.05 to 1.95$)$ for the first trimester, 1.50 (1.08 to 2.09) for the second trimester, and 1.43 (1.03 to 1.98) for the third trimester (table 2 and fig 2).

Results differed slightly when antidepressants were broken down into SSRIs and non-SSRIs. The hazard ratios for both were similar in the crude estimate and model 1 but different in models 2 and 3. In model 3, the adjusted hazard ratio for SSRIs was 1.11 (95\% confidence interval 0.77 to 1.60 ), whereas for non-SSRIs it was 1.59 (1.19 to 2.14). Table 2 and figure 2 summarise the results.

\section{Comparisons}

Preconception users (negative control group 1) versus never users -in model 3 the crude hazard ratio when preconception users of antidepressants were compared with never users was 2.91 and the adjusted hazard ratio was 1.76 (95\% confidence interval 1.36 to 2.30). The risk of ADHD in offspring was statistically significantly increased in preconception users (table 3 and fig 3).

Never users with psychiatric disorders (negative control group 2) versus never users without psychiatric disorders-when the analysis was restricted to mothers who had never used antidepressants or antipsychotics, the risk of ADHD in offspring was higher in mothers with psychiatric disorders. The fully adjusted hazard ratio for psychiatric disorders before pregnancy was 1.94 (95\% confidence interval 1.61 to 2.35 ) and during pregnancy was 1.84 (1.54 to 2.18). The risk estimates were similar to those for both preconception users and current users (table 3 and fig 3).

Gestational use of antidepressants versus antipsychotics (active comparator group)-gestational use of antidepressants yielded a crude hazard ratio of 1.15 for risk of ADHD in offspring compared with prenatal use of antipsychotic (fully adjusted hazard ratio 1.27, 95\% confidence interval 0.73 to 2.18). The comparison between use of SSRI and non-SSRI antidepressants showed no statistically significant difference between the two drug classes, with a crude hazard ratio of 0.75 and fully adjusted hazard ratio of 0.70 (95\% confidence interval 0.38 to 1.29) for risk of ADHD (table 3 and fig 4).

Gestational use of antidepressants versus preconception users-in model 3 the crude hazard ratio when gestational users of antidepressants were compared with preconception users was 0.81 and the adjusted hazard ratio was 0.75 (95\% confidence interval 0.51 to 1.10). There was no statistically significant difference in the risk of ADHD in offspring between gestational users of antidepressants and preconception users (table 3 and fig 4).

\section{Sensitivity analyses}

All other sensitivity analyses returned results similar to those of the main analyses (see supplementary material 3 to 8$)$. 


\begin{tabular}{|c|c|c|c|c|}
\hline Characteristics & $\begin{array}{l}\text { Antidepressants } \\
(\mathrm{n}=1024)\end{array}$ & $\begin{array}{l}\text { Antipsychotics } \\
(\mathrm{n}=364)\end{array}$ & $\begin{array}{l}\text { Antidepressants } \\
\text { and antipsychotics } \\
(\mathrm{n}=228)\end{array}$ & $\begin{array}{l}\text { No antidepressants } \\
\text { or antipsychotics } \\
\text { ( } n=189002)\end{array}$ \\
\hline \multicolumn{5}{|l|}{ Children } \\
\hline Mean (SD) follow-up time (patient years) & $8.9(2.2)$ & $9.5(2.4)$ & $8.9(2.2)$ & $9.3(2.2)$ \\
\hline Attention-deficit/hyperactivity disorder & $58(5.7)$ & $21(5.8)$ & $16(7.0)$ & $5564(2.9)$ \\
\hline Boy & $552(53.9)$ & $192(52.8)$ & $122(53.5)$ & $98316(52.0)$ \\
\hline Spontaneous vaginal delivery & $670(65.4)$ & $233(64.0)$ & $136(59.7)$ & $123834(65.5)$ \\
\hline Multiple pregnancy & $22(2.1)$ & $4(1.1)$ & $4(1.8)$ & $5466(2.9)$ \\
\hline Birth trauma & $4(0.4)$ & $1(0.3)$ & $1(0.4)$ & $862(0.5)$ \\
\hline \multicolumn{5}{|l|}{ Timing of Apgar score $<7$ : } \\
\hline 1 minute & $44(4.3)$ & $22(6.0)$ & $22(9.6)$ & $6983(3.7)$ \\
\hline 5 minutes & $3(0.3)$ & $1(0.3)$ & $3(1.3)$ & $591(0.3)$ \\
\hline \multicolumn{5}{|l|}{ Birth weight (g): } \\
\hline$<1500$ & $13(1.3)$ & $7(1.9)$ & $4(1.8)$ & $2213(1.2)$ \\
\hline $1500-2499$ & $92(9.0)$ & $28(7.7)$ & $26(11.4)$ & $14291(7.6)$ \\
\hline$\geq 2500$ & $919(89.7)$ & $329(90.4)$ & $198(86.8)$ & 172498 (91.3) \\
\hline \multicolumn{5}{|l|}{ Gestation weeks: } \\
\hline$<33$ & $17(1.7)$ & $12(3.3)$ & $5(2.2)$ & $3502(1.9)$ \\
\hline $33-36$ & $95(9.3)$ & $32(8.8)$ & $29(12.7)$ & $12753(6.8)$ \\
\hline$>36$ & $912(89.1)$ & $320(87.9)$ & $194(85.1)$ & $172747(91.3)$ \\
\hline \multicolumn{5}{|l|}{ Mothers } \\
\hline Mean (SD) maternal age at delivery (years) & $32.3(5.53)$ & $31.7(5.60)$ & $32.3(6.05)$ & $31.2(5.10)$ \\
\hline \multicolumn{5}{|l|}{ Maternal underlying conditions: } \\
\hline Epilepsy & $6(0.6)$ & $1(0.3)$ & $1(0.4)$ & $299(0.16)$ \\
\hline Diabetes mellitus before pregnancy & $9(0.9)$ & $2(0.6)$ & $3(1.3)$ & $444(0.2)$ \\
\hline Gestational diabetes mellitus & $45(4.4)$ & $26(7.1)$ & $16(7.0)$ & $5112(2.7)$ \\
\hline Hypertension & $43(4.2)$ & $17(4.7)$ & $10(4.4)$ & $6393(3.4)$ \\
\hline Psychiatric illness & $713(69.6)$ & $308(84.6)$ & $185(81.1)$ & $2978(1.6)$ \\
\hline \multicolumn{5}{|l|}{ Use of psychiatric drugs prepregnancy: } \\
\hline Antidepressants & $612(59.8)$ & $56(15.4)$ & $177(77.6)$ & $1430(0.8)$ \\
\hline Antipsychotics & $95(9.3)$ & $287(78.9)$ & $161(70.6)$ & $445(0.2)$ \\
\hline \multicolumn{5}{|l|}{ Parity: } \\
\hline 0 & $328(32.0)$ & $183(50.3)$ & $92(40.4)$ & $97599(51.6)$ \\
\hline 1 & $425(41.5)$ & $102(28.0)$ & $76(33.3)$ & $70962(37.6)$ \\
\hline 2 & $182(17.8)$ & $51(14.0)$ & $43(18.9)$ & $16023(8.5)$ \\
\hline$\geq 3$ & $89(8.7)$ & $28(7.7)$ & $17(7.5)$ & $4418(2.3)$ \\
\hline \multicolumn{5}{|l|}{ Median household income (HK\$) } \\
\hline$<19300$ & $199(19.4)$ & $93(25.6)$ & $39(17.1)$ & $37828(20.0)$ \\
\hline 19300-21999 & $376(36.7)$ & $117(32.1)$ & 85 (37.3) & $54675(28.9)$ \\
\hline $22000-25999$ & $226(22.1)$ & $88(24.2)$ & $56(24.6)$ & $46018(24.4)$ \\
\hline$\geq 26000$ & $223(21.8)$ & $66(18.1)$ & $48(21.1)$ & $50481(26.7)$ \\
\hline
\end{tabular}

\section{Sibling matched analysis}

The sibling matched analysis included 53616 children of 26049 mothers. In this subgroup, 1510 children had ADHD. There was no statistically significant difference in the risk of ADHD in siblings of mothers who were gestational users of antidepressants compared with non-gestational users (adjusted hazard ratio 0.54, 0.17 to 1.74 ).

\section{Discussion}

This cohort study of more than 190000 mother-child pairs in the Hong Kong Clinical Data Analysis and Reporting System (CDARS) database, found that prenatal use of antidepressants is associated with an increased risk of ADHD. The risk of ADHD was, however, similarly increased for preconception use of antidepressants (negative control group 1) and in children of mothers with psychiatric disorders who had not used antidepressants (negative control group 2). Furthermore, direct comparisons between the gestational user group and an active antipsychotic comparator group, the combined negative control groups 1 and 2, and the sibling matched non-gestational user group yielded statistically non-significant results.

\section{Comparison with other studies}

Most of the published population based studies, with the exception of Castro et al, ${ }^{8}$ reported similar results for risk of ADHD with prenatal use of antidepressants, ranging from 1.16 to 1.81 . $^{6-11}$ Preconception use of antidepressants (negative control group 1) is unlikely to contribute to intrauterine exposure and thus it is not plausible that such use is causally associated with ADHD in offspring. Based on the observed positive association between the negative control groups and the increased risk of ADHD in offspring, it is likely that the 


\begin{tabular}{|c|c|c|c|c|c|c|c|c|}
\hline \multirow[b]{2}{*}{ Exposures } & \multicolumn{2}{|l|}{ Crude estimate } & \multicolumn{2}{|l|}{ Model 1* } & \multicolumn{2}{|l|}{ Model $2 \dagger$} & \multicolumn{2}{|l|}{ Model 3¥ } \\
\hline & $\begin{array}{l}\text { Hazard ratio } \\
(95 \% \mathrm{Cl})\end{array}$ & $P$ value & $\begin{array}{l}\text { Hazard ratio } \\
(95 \% \mathrm{Cl})\end{array}$ & $P$ value & $\begin{array}{l}\text { Hazard ratio } \\
(95 \% \mathrm{Cl})\end{array}$ & $P$ value & $\begin{array}{l}\text { Hazard ratio } \\
(95 \% \mathrm{Cl})\end{array}$ & P value \\
\hline \multicolumn{9}{|l|}{ Antidepressants§: } \\
\hline During pregnancy & $2.26(1.80$ to 2.84$)$ & $<0.01$ & 2.39 (1.90 to 3.02$)$ & $<0.01$ & 1.41 (1.08 to 1.83$)$ & 0.01 & 1.39 (1.07 to 1.82$)$ & 0.01 \\
\hline 1st trimester & 2.29 (1.74 to 3.03$)$ & $<0.01$ & 2.45 (1.85 to 3.25$)$ & $<0.01$ & 1.48 (1.09 to 2.00$)$ & 0.01 & $1.43(1.05$ to 1.95$)$ & 0.02 \\
\hline 2nd trimester & 2.41 (1.78 to 3.26$)$ & $<0.01$ & 2.65 (1.96 to 3.59$)$ & $<0.01$ & 1.51 (1.09 to 2.10$)$ & 0.01 & 1.50 (1.08 to 2.09$)$ & 0.02 \\
\hline 3rd trimester & 2.37 (1.76 to 3.17$)$ & $<0.01$ & 2.55 (1.90 to 3.42$)$ & $<0.01$ & 1.40 (1.01 to 1.93$)$ & 0.04 & 1.43 (1.03 to 1.98$)$ & 0.03 \\
\hline \multicolumn{9}{|l|}{ SSRISף: } \\
\hline During pregnancy & 2.17 (1.56 to 3.03 ) & $<0.01$ & 2.23 (1.60 to 3.12 ) & $<0.01$ & 1.23 (0.86 to 1.77$)$ & 0.26 & 1.11 (0.77 to 1.60$)$ & 0.57 \\
\hline 1st trimester & 2.21 (1.49 to 3.27 ) & $<0.01$ & 2.32 (1.57 to 3.45$)$ & $<0.01$ & 1.31 (0.86 to 1.98$)$ & 0.21 & $1.18(0.78$ to 1.80$)$ & 0.43 \\
\hline 2nd trimester & 2.11 (1.31 to 3.41$)$ & $<0.01$ & $2.22(1.37$ to 3.60$)$ & $<0.01$ & $1.20(0.72$ to 1.98$)$ & 0.48 & 1.10 (0.66 to 1.83$)$ & 0.71 \\
\hline 3rd trimester & 2.21 (1.38 to 3.50$)$ & $<0.01$ & 2.26 (1.41 to 3.63 ) & $<0.01$ & 1.19 (0.73 to 1.93 ) & 0.48 & 1.16 (0.71 to 1.88 ) & 0.56 \\
\hline \multicolumn{9}{|l|}{ Non-SSRIs ${ }^{* *}$} \\
\hline During pregnancy & 2.56 (1.95 to 3.35$)$ & $<0.01$ & $2.76(2.10$ to 3.62$)$ & $<0.01$ & $1.63(1.22$ to 2.19$)$ & $<0.01$ & 1.59 (1.19 to 2.14$)$ & $<0.01$ \\
\hline 1st trimester & $2.57(1.82$ to 3.64$)$ & $<0.01$ & 2.80 (1.96 to 3.99$)$ & $<0.01$ & $1.73(1.20$ to 2.49$)$ & $<0.01$ & 1.64 (1.13 to 2.38$)$ & $<0.01$ \\
\hline 2nd trimester & 2.70 (1.88 to 3.86$)$ & $<0.01$ & 3.04 (2.12 to 4.38$)$ & $<0.01$ & 1.75 (1.19 to 2.55$)$ & $<0.01$ & $1.72(1.16$ to 2.53$)$ & $<0.01$ \\
\hline 3rd trimester & 2.70 (1.91 to 3.83$)$ & $<0.01$ & 2.96 (2.08 to 4.20$)$ & $<0.01$ & 1.62 (1.12 to 2.35$)$ & 0.01 & 1.65 (1.13 to 2.40$)$ & $<0.01$ \\
\hline
\end{tabular}

SSRI=selective serotonin reuptake inhibitor.

*Adjusted for maternal age at delivery, infant’s sex, birth year, birth hospital, parity, maternal underlying illness before delivery (pre-existing diabetes, epilepsy, gestational diabetes,

hypertension), and socioeconomic status.

tAdjusted for all factors in model 1 and maternal psychiatric conditions.

$\ddagger$ Adjusted for all factors in model 2 and other psychiatric drug use.

§ADHD cases: 74 in antidepressant users, 5564 in non-users.

IADHD cases: 18 in SSRI users, 5564 in non-users.

${ }^{*}$ ADHD cases: 31 in non-SSRI users, 5564 in non-users.

increased risk from prenatal use might at least be partially explained by confounding due to pre-existing conditions of the mothers. ADHD is highly heritable, ${ }^{2122}$ and parents of children with ADHD are themselves more likely to have the same or related mental disorders. In recent years it has become apparent that although ADHD often persists into adulthood and is associated with high levels of psychiatric comorbidity, including increased rates of depression and anxiety, many adults with ADHD never receive a proper diagnosis or

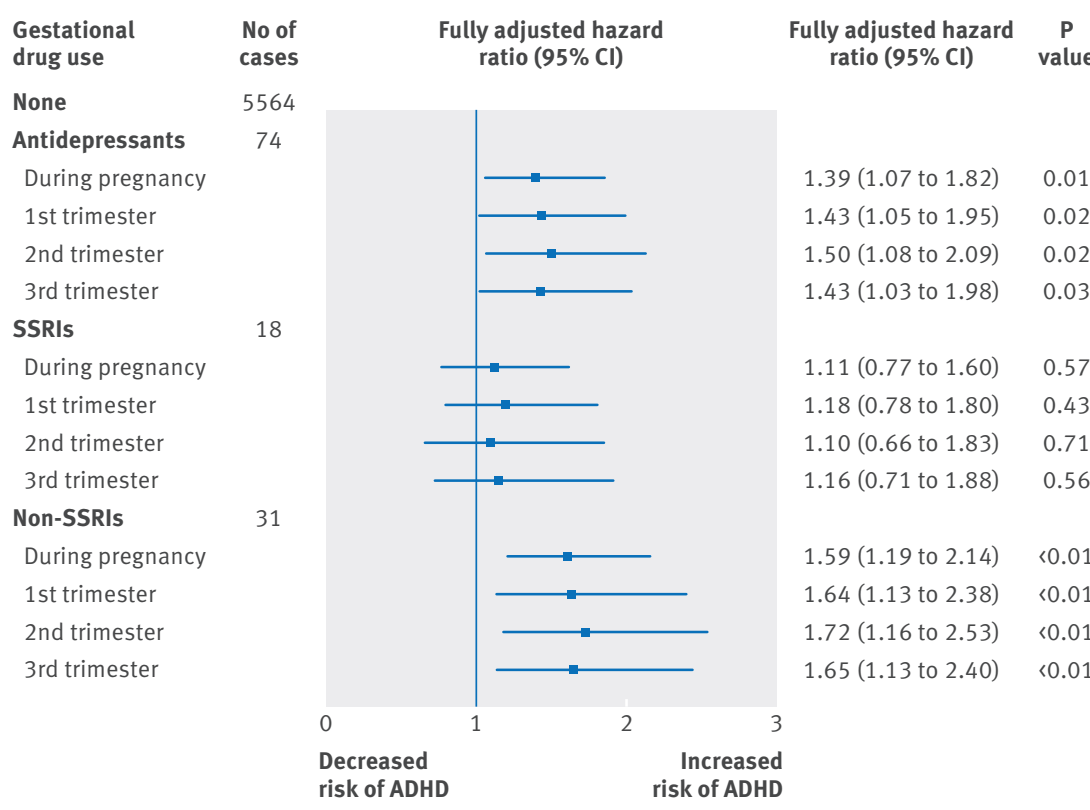

Fig 2 | Fully adjusted results from analysis for gestational users of antidepressants compared with non-gestational users. SSRI=selective serotonin reuptake inhibitor; $\mathrm{ADHD}=$ attention-deficit/hyperactivity disorder treatment. ${ }^{23}$ This could explain why we found a possible link between preconception use, psychiatric disorders in mothers, and ADHD in children. Indeed, this study also found that mothers in both negative control groups are at similar increased risk of having offspring with ADHD as the mothers in the gestational treatment group. As psychiatric diseases are the main indication for prescription of antidepressants, our results suggest the possibility of confounding by indication.

About $0.7 \%$ of children (1252 out of 190618) in our cohort were exposed to antidepressant prenatally, which is slightly lower than in previous studies. -10 $^{-10}$ Comparing standardised differences in the rate of antidepressant use between the current study and previous studies (see supplementary material 9), our rate is lower than one study only ${ }^{7}$ and comparable with five other studies. ${ }^{6-11}$ In a previous drug prevalence study in Hong Kong, the prescribing prevalence was generally lower than in Western countries. ${ }^{24}$ This may be explained by the conservative approach in local practice. ${ }^{2425}$ Nevertheless, there is no reason why these differences in rates of antidepressant use should alter the conclusions of the study.

Many previous studies did not explore class and drug specific associations. ${ }^{6-11}$ In the current study we identified a slightly different result in our subgroup analysis on SSRIs and non-SSRIs, with no statistically significant association between prenatal use of SSRIs and risk of ADHD in children, although we cannot exclude the possibility of type 2 error. However, the difference in results between the SSRI and non-SSRI group may suggest the potential effect of disease severity rather than a differential effect of drug class. SSRIs are usually the first line treatment and thus mothers who use non-SSRIs during pregnancy may have more severe psychiatric conditions. 


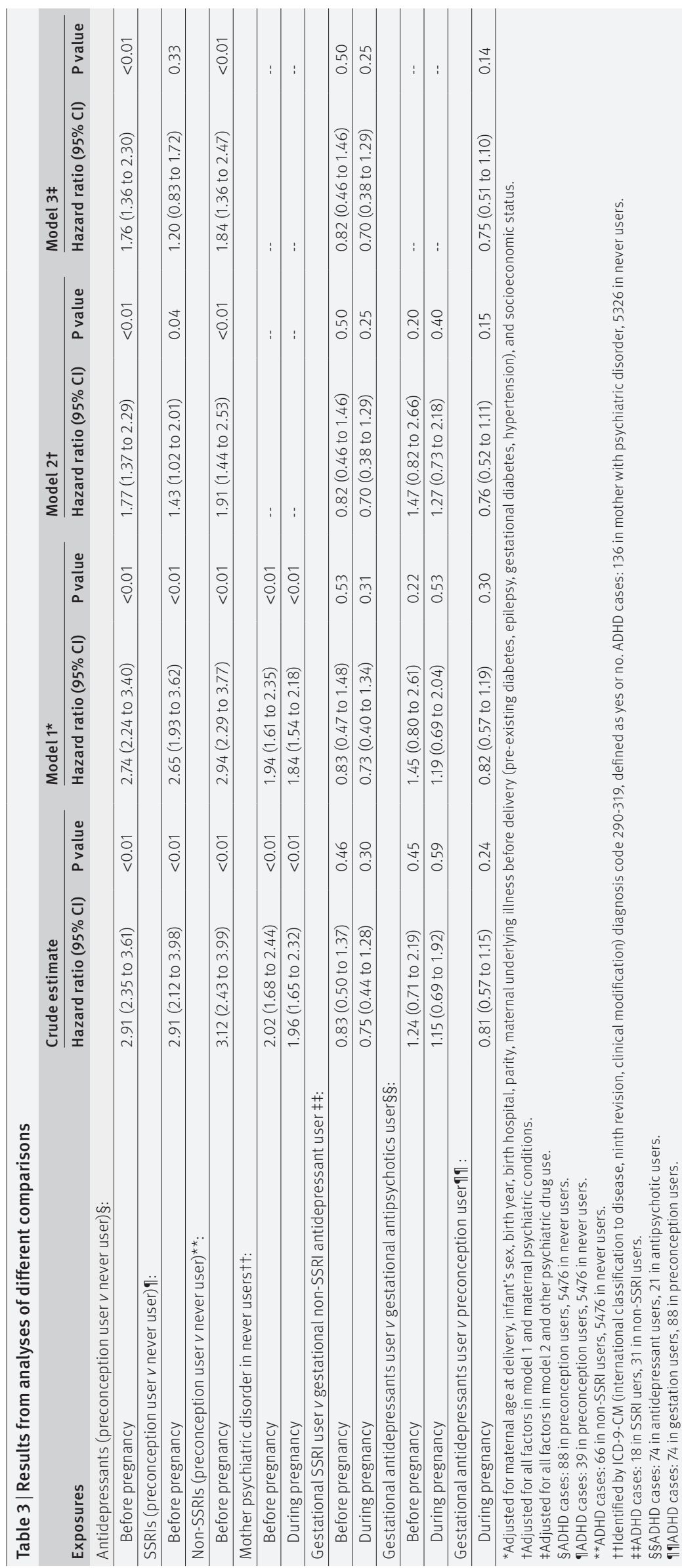

This might explain the difference in the result, although further studies should evaluate if the corresponding risk differs between SSRI and non-SSRI antidepressants.

The results of one study ${ }^{8}$ differed from those of previous studies even though this was a replication study using the same data source and similar methods. ${ }^{10}$ The study reported no statistically significant increase in risk associated with prenatal use of antidepressants but did find an increased risk associated with prepregnancy antidepressant use. The authors suggest that it is therefore possible that the association between antidepressant use and ADHD in offspring might be indicative of confounding by indication. It is, however, difficult to explain why confounding by indication should only occur for prepregnancy antidepressant use and not prenatal use. In contrast, our study has shown consistent results for gestational users (active treatment group) in the three trimesters, preconception users (negative control group 1), and never users with a psychiatric diagnosis (negative control group 2). Our study therefore provides stronger evidence to support the possibility that the observed associations might represent confounding by indication.

\section{Strengths and limitations of this study}

The novelty of the current study is that we utilised two complementary planned negative control groups. An increased risk of ADHD was observed in children whose mothers had used antidepressants before conception (negative control group 1) and those mothers with a psychiatric diagnosis who had never used antidepressants (negative control group 2). Although it is difficult to adjust for confounding by indication in the classic population based analysis, the outcomes of the two negative control analyses aid the interpretation of our results. To further explore the possibility of confounding by indication, we preselected mothers who used antipsychotic drugs as an active comparator group, and the lack of association in this analysis adds further weight to the interpretation. Finally, we also conducted a sibling matched analysis using sibling pairs. Although we cannot exclude the possibility of type 2 error in this analysis, the results further strengthen our hypothesis that confounding by indication may play a major role in the observed positive association between gestational use of antidepressants and ADHD in offspring. We have not been able to identify any other published studies that have applied such a comprehensive series of analyses to these associations. We recommend that this approach should be considered in future studies that investigate gestational drug use and outcome in offspring, particularly diseases and disorders with high heritability and that are therefore more likely to be confounded by indication.

Previous studies have been based in Scandinavian countries and North America, countries with a large population of white people. Our study is the first based in Asia. After adjusting for confounding factors, our comprehensive analyses in a predominately Chinese population yielded similar results to a recently published Swedish study using population based and sibling based analyses. Both studies do not support gestational antidepressant use as significantly 


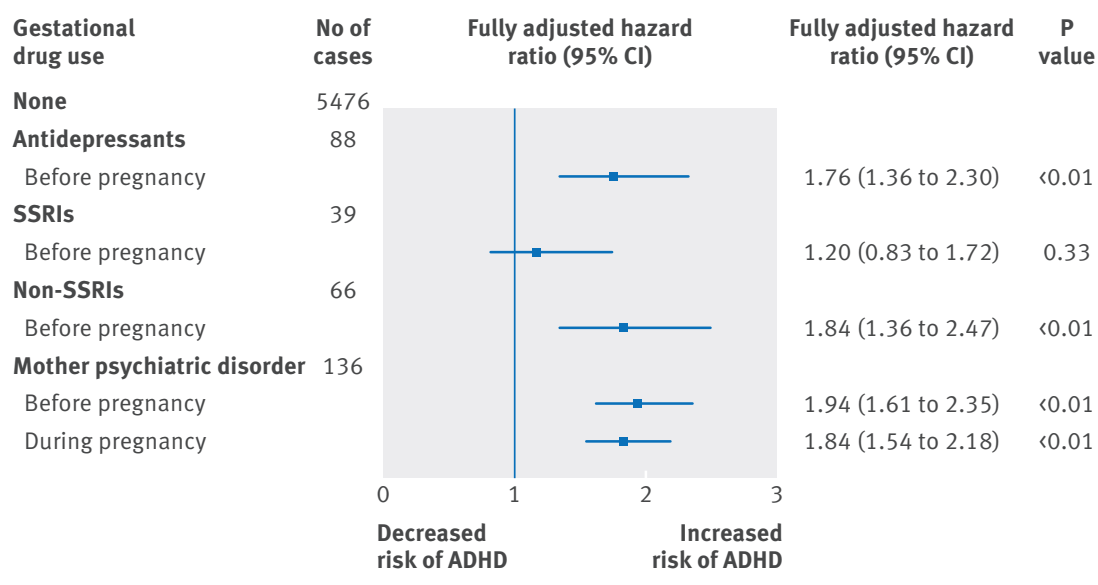

Fig 3 | Fully adjusted results from analyses of different comparisons. SSRI=selective serotonin reuptake inhibitor; $A D H D=$ attention-deficit/hyperactivity disorder

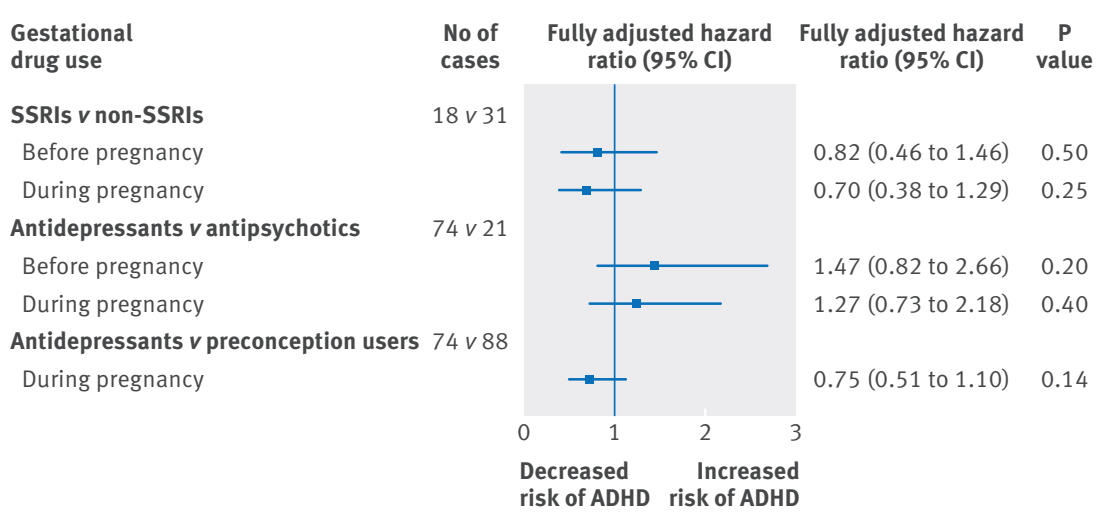

Fig 4 Fully adjusted results from analyses of comparisons with active comparator.

SSRI=selective serotonin reuptake inhibitor; $A D H D=$ attention-deficit/hyperactivity disorder

increasing the risk of ADHD in offspring. As the two study populations have a different genetic composition, healthcare systems, and environments, this suggests the findings are broadly generalisable to other populations.

This study also has several important weaknesses. A limitation of register based studies is that they only include cases that are clinically detected. In our cohort, about 3\% of children had a diagnosis of ADHD or used ADHD treatment, which is lower than the prevalence estimates from epidemiological studies (5-7\%). However, it is generally accepted that for most non-US countries the administrative prevalence (number of ADHD cases that are diagnosed and treated) is somewhat lower than the epidemiological prevalence. ${ }^{26}$ Under-diagnosis of less severe cases of ADHD is likely to be the same across the groups; hence it is unlikely to have affected the findings or conclusions of the current study.

CDARS only contains information from publicly funded healthcare medical records and therefore does not include data from private medical practitioners or hospitals. Based on the birth statistics in Hong Kong, about 45000 live births occurred in 2009. ${ }^{27}$ Our data captured more than 31000 birth events in the same year, thus including about $70 \%$ of births in Hong Kong. ${ }^{27}$ Notably, the public sector is the main provider of specialist care for neurodevelopmental disorders in Hong Kong. ${ }^{28}$ Children with neurodevelopmental disorders usually require comprehensive long term treatment and monitoring, hence they are usually under the care of publicly funded healthcare. It is therefore likely that most of the children who received a diagnosis of ADHD will have been included in this study.

As antidepressant use is defined by prescriptions written by clinicians and dispensed by pharmacies, it is unclear whether the drugs were actually taken by women in accordance with information on the prescription (adherence)-that is, use of the drugs might have been longer than the intended prescribing period. We addressed usage misclassification by conducting a duration-outcome analysis, adding 30 and 60 days after the end of the expected period for antidepressant use. However, we could not exclude the possibility that included mothers might obtain prescriptions for antidepressant from the private sector, which we would not be able to identify; although the public sector is by far the main provider of specialist care in Hong Kong. Thus the potential for missing drug records is minimal. Drug usage misclassification will bias the risk estimate towards the null. We may potentially underestimate the risk but this would not affect the conclusions of the study.

\section{Policy implications}

Since the first report of a possible association between in utero exposure to antidepressants and neurodevelopmental disorders in children, both patients and clinicians have faced the dilemma of how to manage women with severe affective disorders both when they are trying to conceive and when they are pregnant. Major adverse effects can occur when stopping drugs abruptly or withholding antidepressants during pregnancy. The present findings provide useful data to help guide clinicians in decision making.

\section{Conclusions}

Previous reports might have overestimated the association between gestational use of antidepressants and ADHD in offspring because they have failed to control for shared family factors. Although we cannot completely discount the possibility that gestational use of antidepressants is a causal factor, our findings raise the possibility that confounding by indication might at least partially explain the observed association. We propose that if a causal association exists, then the size of the effect is probably smaller than that previously reported. However, decision making about antidepressant use in pregnancy remains important and requires an assessment of the risks and benefits in the context of the individual woman and family.

\section{AUTHOR AFFILIATIONS}

${ }^{1}$ Centre for Safe Medication Practice and Research, Department of Pharmacology and Pharmacy, Li Ka Shing Faculty of Medicine, The University of Hong Kong, Hong Kong, China

2Department of Paediatrics and Adolescent Medicine, Li Ka Shing Faculty of Medicine, The University of Hong Kong, Hong Kong, China

${ }^{3}$ Centre for Paediatric Pharmacy Research, Research Department of Practice and Policy, UCL School of Pharmacy, London, UK 
${ }^{4}$ Department of Medical Informatics, Erasmus University Medical Center, Rotterdam, Netherlands

${ }^{5}$ Division of Neuroscience, Medical Research Institute, University of Dundee, Dundee, UK

${ }^{6}$ Departments of Paediatrics and Psychiatry, Faculty of Medicine, Dentistry and Health Sciences, University of Melbourne, Melbourne, Australia

${ }^{7}$ King's College London, Institute of Psychiatry and NIHR Biomedical Research Centre for Mental Health, London, UK

${ }^{8}$ Department of Psychiatry, Queen Mary Hospital, Hong Kong, China

${ }^{9}$ Janssen Research and Development, LLC, Titusville, NJ, USA

We thank the Hong Kong Hospital Authority for access to the data from CDARS and Lisa Wong for help with the manuscript.

Contributors: KKCM and ICKW had the original idea for the study, contributed to the development of the study, extracted data from the source database, constructed the study design and the statistical model, reviewed the literature, and act as guarantors for the study. KKCM and WCYL undertook the statistical analysis. KKCM wrote the first draft of the manuscript. PI and PKLC validated the diagnosis codes from the database. ICKW is the principal investigator and provided oversight for all aspects of this project. MCJMS and MJS provided critical input to the analyses and design. All authors contributed to the interpretation of the analysis, critically reviewed and revised the manuscript, and approved the final manuscript as submitted. Funding: None.

Competing interests: All authors have completed the ICMJE uniform disclosure form at www.icmje.org/coi_disclosure.pdf and declare: EWC reports grants from Janssen (a division of Johnson \& Johnson), BMS, Pfizer, the Research Grants Council (RGC, Hong Kong), received for other work. DC reports grants from the European Union FP7 programme and Shire and honorariums from Shire, Eli Lilly, Novartis, and Janssen-Cilag; acted as an advisor to Shire and Lundbeck; and received royalties from Oxford University press. DC was a member of the British Association for Psychopharmacology ADHD, Depression and Bipolar Disorder guideline groups. ES reports grants from Innovative Medicines Initiative (IMI), National Institute for Health Research programme grant for applied research, and the National Institute for Health Research Biomedical Research Centre for Mental Health. PI reports grants from RGC and the Health and Medical Research Fund (Food and Health Bureau, Hong Kong). ICKW reports grants from RGC, IMI, Shire, Janssen-Cilag, Eli-Lily, Pfizer, and the European Union FP7 programme, outside the submitted work. ICKW is a member of the National Institute for Health and Clinical Excellence ADHD Guideline Group and was a member of the British Association for Psychopharmacology ADHD guideline group and acted as an advisor to Shire. PKLC acted as an advisor to Eli Lilly. MCJMS is leading a research group that received grants for specific postauthorisation safety projects from Novartis, Boehringer, GSK, and Servier, none related to this topic. MJS is a full time employee and shareholder of Johnson \& Johnson.

Ethical approval: This study protocol was approved by the institutional review board of the University of Hong Kong/Hospital Authority Hong Kong West Cluster (reference No UW15-086).

Data sharing: No additional data available.

Transparency: The manuscript's guarantors (KM and IW) affirm that the manuscript is an honest, accurate, and transparent account of the study being reported; that no important aspects of the study have been omitted; and that any discrepancies from the study as planned (and, if relevant, registered) have been explained.

This is an Open Access article distributed in accordance with the Creative Commons Attribution Non Commercial (CC BY-NC 4.0) license, which permits others to distribute, remix, adapt, build upon this work non-commercially, and license their derivative works on different terms, provided the original work is properly cited and the use is noncommercial. See: http://creativecommons.org/licenses/by-nc/4.0/.

1 American Psychiatric Association. Diagnostic and Statistical Manual of Mental Disorders.5th ed. American Psychiatric Publishing, 2013.

2 Polanczyk GV, Willcutt EG, Salum GA, Kieling C, Rohde LA. ADHD prevalence estimates across three decades: an updated systematic review and meta-regression analysis. Int J Epidemiol 2014;43:434-42. doi:10.1093/ije/dyt261.

3 Thomas R, Sanders S, Doust J, Beller E, Glasziou P. Prevalence of attentiondeficit/hyperactivity disorder: a systematic review and meta-analysis. Pediatrics 2015;135:e994-1001. doi:10.1542/peds.2014-3482.

4 Karam RG, Breda V, Picon FA, et al. Persistence and remission of ADHD during adulthood: a 7-year clinical follow-up study. Psychol Med 2015:45:2045-56 doi:10.1017/S0033291714003183.
5 Fleck K, Jacob C, Philipsen A, et al. Child impact on family functioning: a multivariate analysis in multiplex families with children and mothers both affected by attention-deficit/hyperactivity disorder (ADHD). Atten Defic Hyperact Disord 2015;7:211-23. doi:10.1007/s12402-014-0164-8.

6 Laugesen K, Olsen MS, Telén Andersen AB, Frøslev T, Sørensen HT. In utero exposure to antidepressant drugs and risk of attention deficit hyperactivity disorder: a nationwide Danish cohort study. BMJ Open 2013;3:e003507. doi:10.1136/bmjopen-2013-003507.

7 Figueroa R. Use of antidepressants during pregnancy and risk of attention-deficit/hyperactivity disorder in the offspring. I Dev Behav Pediatr 2010;31:641-8. doi:10.1097/DBP.0b013e3181e5ac93.

8 Castro VM, Kong SW, Clements CC, et al. Absence of evidence for increase in risk for autism or attention-deficit hyperactivity disorde following antidepressant exposure during pregnancy: a replication study. Transl Psychiatry 2016;6:e708. doi:10.1038/tp.2015.190.

9 Malm H, Brown AS, Gissler M, et al. Gestational Exposure to Selective Serotonin Reuptake Inhibitors and Offspring Psychiatric Disorders: A National Register-Based Study. J Am Acad Child Adolesc Psychiatry 2016;55:359-66. doi:10.1016/j.jaac.2016.02.013.

10 Clements CC, Castro VM, Blumenthal SR, et al. Prenatal antidepressant exposure is associated with risk for attention-deficit hyperactivity disorder but not autism spectrum disorder in a large health system. Mol Psychiatry 2015;20:727-34. doi:10.1038/mp.2014.90.

11 Sujan AC, Rickert ME, Öberg AS, et al. Associations of maternal antidepressant use during the first trimester of pregnancy with preterm birth, small for gestational age, autism spectrum disorder, and attention-deficit/hyperactivity disorder in offspring. JAMA 2017;317:1553-62. doi:10.1001/jama.2017.3413.

12 Daviss WB. A review of co-morbid depression in pediatric ADHD: etiology, phenomenology, and treatment. / Child Adolesc Psychopharmacol 2008;18:565-71. doi:10.1089/cap.2008.032

13 Larsson H, Rydén E, Boman M, Långström N, Lichtenstein P, Landén M. Risk of bipolar disorder and schizophrenia in relatives of people with attention-deficit hyperactivity disorder. Br J Psychiatry 2013;203:103-6. doi:10.1192/bjp.bp.112.120808.

14 Man KKC, Chan EW, Coghill D, et al. Methylphenidate and the risk of trauma. Pediatrics 2015;135:40-8. doi:10.1542/peds.2014-1738.

15 Chui CSL, Man KKC, Cheng CL, et al. An investigation of the potential association between retinal detachment and oral fluoroquinolones: a self-controlled case series study. I Antimicrob Chemother 2014;69:2563-7. doi:10.1093/jac/dku145.

16 Chan EW, Lau WCY, Leung WK, et al. Prevention of DabigatranRelated Gastrointestinal Bleeding With Gastroprotective Agents: A Population-Based Study. Gastroenterology 2015;149:586-95.e3. doi:10.1053/j.gastro.2015.05.002

17 Wong AYS, Wong ICK, Chui CSL, et al. Association Between Acute Neuropsychiatric Events and Helicobacter pylori Therapy Containing Clarithromycin. JAMA Intern Med 2016:176:828-34. doi:10.1001/ jamainternmed.2016.1586.

18 Chui CSL, Chan EW, Wong AYS, Root A, Douglas IJ, Wong ICK. Association between oral fluoroquinolones and seizures: A self-controlled case series study. Neurology 2016;86:1708-15. doi:10.1212/WNL.0000000000002633.

$19 \mathrm{HAHO} / \mathrm{ITD}$. Clinical Data Analysis \& Reporting System (CDARS) User's Manual. In: Authority H, ed. 2.0 edn. Hong Kong: 2003. p3.

20 Obel C, Olsen J, Henriksen TB, et al. Is maternal smoking during pregnancy a risk factor for hyperkinetic disorder?--Findings from a sibling design. Int J Epidemiol 2011:40:338-45. doi:10.1093/ije/dyq185.

21 Ronald A, Simonoff E, Kuntsi J, Asherson P, Plomin R. Evidence for overlapping genetic influences on autistic and ADHD behaviours in a community twin sample. J Child Psychol Psychiatry 2008;49:535-42. doi:10.1111/j.1469-7610.2007.01857.x

22 Smalley SL. Genetic influences in childhood-onset psychiatric disorders: autism and attention-deficit/hyperactivity disorder. Am J Hum Genet 1997:60:1276-82. doi:10.1086/515485.

23 Asherson P, Akehurst R, Kooij JJS, et al. Under diagnosis of adult ADHD: cultural influences and societal burden. J Atten Disord 2012:16(Suppl):20S-38S. doi:10.1177/1087054711435360.

24 Man KK, Ip P, Hsia Y, et al. ADHD Drug Prescribing Trend Is Increasing Among Children and Adolescents in Hong Kong. J Atten Disord 2014; 1087054714536047.

25 Chan EW, Lau WC, Siu CW, et al. Effect of suboptimal anticoagulation treatment with antiplatelet therapy and warfarin on clinical outcomes in patients with nonvalvular atrial fibrillation: A population-wide cohort study. Heart Rhythm 2016;13:1581-8. doi:10.1016/j.hrthm.2016.03.049.

26 Hinshaw SP, Scheffler RM, Fulton BD, et al. International variation in treatment procedures for ADHD: social context and recent trends. Psychiatr Serv 2011;62:459-64. doi:10.1176/ps.62.5.pss6205 0459.

27 Census \& Statistics Department HG. Hong Kong Monthly Digest of Statistics: The Fertility Trend in Hong Kong, 1981 to 2012, 2013.

28 Leung GM, Tin KYK, O'Donnell O. Redistribution or horizontal equity in Hong Kong's mixed public-private health system: a policy conundrum. Health Econ 2009:18:37-54 doi:10.1002/hec.1342.

Supplementary information: material 1-9 